\title{
Therapeutic targeting of PFKFB3 and PFKFB4 in multiple myeloma cells under hypoxic conditions
}

Seiichi Okabe ( $\nabla$ okabe@tokyo-med.ac.jp )

Tokyo Medical University https://orcid.org/0000-0002-2727-658X

\section{Yuko Tanaka}

Tokyo Medical University: Tokyo Ika Daigaku

Akihiko Gotoh

Tokyo Medical University: Tokyo Ika Daigaku

\section{Research}

Keywords: multiple myeloma, hypoxia, proteasome inhibitor, PFKFB

Posted Date: November 22nd, 2021

DOI: https://doi.org/10.21203/rs.3.rs-1091276/v1

License: (1) This work is licensed under a Creative Commons Attribution 4.0 International License.

Read Full License 


\section{Abstract}

\section{Background}

Multiple myeloma ( $\mathrm{MM})$ is a hematological malignancy characterized by the clonal expansion of plasma cells in the bone marrow. The treatment of MM patients has been dramatically changed by new agents, such as proteasome inhibitors and immunomodulatory drugs; however, many patients will relapse, even if the new agents provide therapeutic advantages. Hypoxia is an important component of the bone-marrow microenvironment. 6-Phosphofructo-2-kinase/fructose-2,6-bisphosphatase (PFKFB) is responsible for maintaining cellular levels of fructose-2,6-bisphosphate, which regulates glycolysis.

Methods

In this study, we investigated the PFKFB functions in myeloma cells under hypoxic conditions. We also investigated whether PFKFB inhibitors could suppress myeloma cells and enhance their sensitivity to proteasome inhibition.

Results

We first investigated the expression of PFKFBs in the myeloma cell lines under hypoxic conditions. Using public microarray datasets (GSE80140 and GSE80545), we found that the gene expressions of PFKFB3 and PFKFB4 were elevated under hypoxic conditions. Hypoxia-inducible factor $1 \mathrm{a}(\mathrm{HIF} 1 \mathrm{a})$ was increased, and the phosphorylation of p38 mitogen-activated protein kinase (p38 MAPK) was activated. Under hypoxia, the activity of proteasome inhibitors was reduced. The PFKFB3 inhibitor, PFK158 and PFKFB4 inhibitor, 5MPN treatment were found to inhibit the growth of myeloma cells. The combined treatment of myeloma cells with carfilzomib and PFK158 or 5MPN was more cytotoxic than each drug alone. Caspase $3 / 7$ activity and cellular cytotoxicity were also increased. In addition, we found that proteasomal activity was reduced by carfilzomib and PFK158 or 5MPN treatment. Intracellular adenosine triphosphate (ATP) levels drastically decreased after combined treatment. The combined treatment also changed the mitochondrial membrane potential in cell death and was effective on the bortezomib-resistant cell line.

Conclusion

PFKFB3 and PFKFB4 are enhanced in hypoxic conditions and are involved in proteasome-inhibitor sensitivity. Our data also suggested that administration of PFKFB3 and PFKFB4 inhibitors may be a powerful strategy against myeloma cells and may enhance the cytotoxic effects of proteasome inhibitors in hypoxic conditions.

\section{Background}

Multiple myeloma (MM) is a malignancy of terminally differentiated plasma cells due to the accumulation of abnormal plasma cells in the bone marrow and monoclonal protein in the serum and/or urine [1]. The plasma cells proliferate in the bone marrow and can result in extensive skeletal destruction 
with osteolytic lesions, osteopenia, and/or pathologic fractures [2]. MM often progresses from a premalignant state called monoclonal gammopathy of undetermined significance (MGUS) [3]. In addition, the risk of MM is much higher in older age groups [4]. Therapeutic strategies for MM have dramatically changed in the last two decades. In clinical settings, symptomatic MM has been treated by new drugs, such as proteasome inhibitors, immunomodulators, and antibody therapies [1]. However, acquisition of resistance causes a relapse of the disease in many myeloma patients, so the disease remains incurable [5]. Thus, the clinical management of MM patients to improve the survival rate of MM remains a challenge.

Bone marrow is considered a tissue with a limited oxygen supply, and one of the hallmarks of the hematopoietic stem cell (HSC) niche is its low oxygen tension [6]. Thus, hypoxia is an important component of the bone marrow microenvironment and the HSC niche. Under conditions of hypoxia, most eukaryotic cells can shift their primary metabolic strategy from predominantly mitochondrial respiration to increased glycolysis to maintain adenosine triphosphate (ATP) levels [7]. Fructose-2,6-bisphosphate accelerates the glycolytic flux by allosterically activating 6-phosphofructo-1-kinase. Fructose-2,6bisphosphate is generated and degraded by the bifunctional enzyme 6-phosphofructo-2-kinase/fructose2,6-bisphosphatase (PFK-2/FBPase-2) [8]. There are four major PFK-2/FBPase-2 isozymes, 6phosphofructo-2-kinase/fructose-2,6-bisphosphatase 1-4 (PFKFB-1-4), which are encoded by four different genes (PFKFB1, PFKFB2, PFKFB3, and PFKFB4) in vertebrates [9]. PFKFB3 and PFKFB4 are cancer-specific isoenzymes of the PFKFB enzyme group [10]. Previous work has demonstrated that one of the PFK-2 isozyme genes, PFKFB3, was induced by hypoxia through the hypoxia-inducible factor-1 (HIF-1) pathway [11]. Metabolites and enzymes can be upregulated or downregulated and may play a crucial role in drug resistance. Hypoxia can not only induce genetic changes but may select for cells that are resistant to apoptosis and thus can survive in the face of accumulating genetic changes [12]. Therefore, we investigated whether hypoxia affects metabolic change in myeloma cells and the activity of the proteasome inhibitor using MM cell lines. We discovered that metabolites and enzymes can be upregulated or downregulated and play a crucial role in drug resistance in myeloma cells.

\section{Methods}

\section{Reagents}

A potent and selective PFKFB3 inhibitor, PFK158, was purchased from MedChemExpress (Monmouth Junction, NJ, USA). A selective inhibitor of PFKFB4, 5-(n-(8-methoxy-4-quinolyl)amino)pentyl nitrate (5MPN) was purchased from Sigma-Aldrich (St. Louis, MO, USA). Carfilzomib was purchased from MedKoo Biosciences (Chapel Hill, NC, USA). Stock solutions of PFK158, 5MPN, and carfilzomib were dissolved in dimethyl sulfoxide. Other reagents were obtained from Sigma-Aldrich.

\section{Cell lines}

The MM cell lines, U266, RPMI8226, MM.1S, and MM.1R, were obtained from the American Type Culture Collection (ATCC, Manassas, VA, USA). The bortezomib-resistant myeloma cell line, KMS-11/BTZ, was 
obtained from the Japanese Collection of Research Bioresources Cell Bank (Ibaraki, Osaka, Japan). These MM cell lines were cultured in Roswell Park Memorial Institute 1640 medium containing 10\% fetal bovine serum (FBS) and $1 \%$ penicillin/streptomycin and maintained at $37^{\circ} \mathrm{C}$ in a $5 \%$ carbon dioxide $\left(\mathrm{CO}_{2}\right)^{-}$ humidified atmosphere. Cell cultures were grown under hypoxic conditions at $37^{\circ} \mathrm{C}$ with an atmosphere of $5 \% \mathrm{CO}_{2}$ and $1 \% \mathrm{O}_{2}$. Experiments were initiated after $72 \mathrm{~h}$ of adaptation to the hypoxic conditions.

\section{Cell proliferation assays}

Cells were treated with PFK158 or 5MPN and/or with carfilzomib for $72 \mathrm{~h}$ and then cell viability was evaluated by trypan blue exclusion or with Cell Counting Kit-8 (Dojindo Laboratories, Mashikimachi, Kumamoto, Japan) followed by measurement of the absorbance at $450 \mathrm{~nm}$. In some experiments, cell cultures were also grown under hypoxic conditions $\left(1 \% \mathrm{O}_{2}\right)$ and treated with PFK158 or 5MPN and/or with carfilzomib. Experiments were initiated after $72 \mathrm{~h}$ of adaptation to the hypoxic conditions. In this study, the Chou-Talalay combination index was used to determine a synergistic (combination index: $\mathrm{Cl}<1)$, additive $(\mathrm{Cl}=1.0)$, or antagonistic $(\mathrm{Cl}>1)$ effect for each combination [13].

\section{Caspase $3 / 7$ activity}

Caspase activity in myeloma cells was examined using the Caspase Glo 3/7 assay kit (Promega, Madison, WI, USA) according to the manufacturer's instructions. After $48 \mathrm{~h}$ of carfilzomib and/or PFK158 or 5MPN treatment, the luminescence of each sample was measured using the EnSpire Multimode Plate Reader (PerkinElmer, Waltham, MA, USA).

\section{Enzyme linked immunosorbent assay (ELISA)}

To investigate phospho p38 MAPK or phospho NF-KB activity, MM cells were cultured in RPMI medium with or without hypoxic conditions. After 2 or $6 \mathrm{~h}$, the cells were harvested and stored at $-80^{\circ} \mathrm{C}$. In some experiments, the hypoxia-inducible factor a (HIFa) inhibitor, FM19G11 (Sigma-Aldrich), or the p38 MAPK inhibitor, SB203580 (Sigma-Aldrich), were used. P38 MAPK phosphorylation was measured using the p38 MAPK (Phospho) [pT180/pY182] Multispecies InstantOne ${ }^{\mathrm{TM}}$ ELISA Kit, and phospho NF-KB was measured using the NF-kB p65 (Phospho) [pS536] Human InstantOne ${ }^{\mathrm{TM}}$ ELISA Kit (Thermo Fisher Scientific, Waltham, MA, USA). Intracellular glucose and lactate dehydrogenase (LDH) release were analyzed using the Glucose Assay Kit-WST (Dojindo) and the LDH-Glo ${ }^{\text {TM }}$ Cytotoxicity Assay (Promega) or the Cytotoxicity LDH Assay kit with water-soluble tetrazolium [WST] salt (Dojindo). ELISA was analyzed using an EnSpire Multimode Plate Reader. All of the measurements were performed in triplicate.

\section{Proteasome activity}

The activity of the 20S proteasome was determined using the 20S Proteasome Assay Kit (Cayman Chemical, Ann Arbor, MI, USA). The cells were treated with the indicated concentrations of carfilzomib and/or PFK158 or 5MPN under hypoxia for $24 \mathrm{~h}$. After the cells were harvested, the proteasome activity 
was analyzed according to the manufacturer's instructions. Fluorescence intensity was measured using the EnSpire Multimode Plate Reader.

\section{Analysis of the mitochondrial membrane potential}

The mitochondrial membrane potential was analyzed using the cationic JC-1 dye and the Mitochondria Staining Kit (Sigma-Aldrich) according to the manufacturer's instructions. After harvest of MM cells, JC-1 monomers and JC-1 aggregates were measured using the EnSpire Multimode Plate Reader.

\section{Quantitative real-time reverse transcription-polymerase chain reaction analysis (RT-PCR)}

Total RNA was extracted from myeloma samples using the RNAqueous®-4PCR Kit (Life Technologies Japan Ltd., Minato-ku, Tokyo, Japan) and reverse transcribed using the First-Strand cDNA Synthesis Kit (OriGene Technologies, Rockville, MD, USA). RT-PCR was performed using the Roche Light Cyber 2.0 detection system (Roche Diagnostic Gmbh, Minato-ku, Tokyo, Japan). The expressions of human PFKFB1, PFKFB2, PFKFB3, PFKFB4, GAPDH, and $\beta$-actin were quantitated using the SYBR Green PCR Kit (Roche) according to the manufacturer's protocol. Specific PCR primers were obtained from Takara Bio Inc. (Otsu, Shiga, Japan).

\section{Immunoblot analysis}

Immunoblot analysis was performed as described previously $[13,14]$. After incubation, the cells were washed twice with ice-cold phosphate-buffered saline and lysed using a radioimmunoprecipitation assay lysis buffer (Merck Millipore, Darmstadt, Germany).

The protein content was quantified using the DC Protein Assay Kit (BioRad, Hercules, CA, USA) according to the manufacturer's instructions. Total cellular proteins $(40 \mu \mathrm{g})$ were separated on $4-20 \%$ polyacrylamide gels (BioRad) and transferred onto polyvinylidene difluoride membranes (Merck KGaA, Darmstadt, Germany). The membranes were probed using appropriate primary antibodies at the appropriate dilutions for $1 \mathrm{~h}$ at room temperature. The blots were visualized by chemiluminescence using the Amersham ECL chemiluminescence kit (GE Healthcare, Chicago, IL, USA). Primary antibodies (Abs) against PFKFB3 and PFKFB4 were purchased from Abcam (Cambridge, UK). NF-KB p65, cleaved caspase 3, cleaved PARP, phospho p38 MAPK, and BCL-2 Abs were purchased from Cell Signaling Technology (Danvers, MA, USA). BCL-XL, p38 MAPK, and $\beta$-actin were purchased from Santa Cruz Biotechnology (Santa Cruz, CA, USA). Abs of HIF1a and BCL2L10 were purchased from GeneTex (Irvine, CA, USA). Three independent experiments were performed in each case.

\section{Statistical analysis}

All presented data were analyzed using Prism 9 (GraphPad Software, San Diego, CA, USA) or Excel software (Microsoft, Redmond, WA, USA). Student's $t$ test was used to determine if the effects on the drug-treated groups were statistically significant compared with the controls. $\mathrm{P}<0.05$ or $\mathrm{P}<0.01$ was considered statistically significant. 


\section{Results}

\section{PFKFB gene expression in myeloma cells under hypoxic conditions}

Tumor cells maintain a high glycolytic rate not only in conditions of adequate oxygen supply but also in hypoxic conditions [16]. Oxygen $\left(\mathrm{O}_{2}\right)$ is a major determinant of cell metabolism and gene expression. In the bone marrow, the measurement of local oxygen tension is quite low. Thus, we first investigated the expressions of metabolic genes in myeloma cells under hypoxia using the Gene Expression Omnibus (GEO) database. We found that the gene expressions of PFKFB3 and PFKFB4 under hypoxic conditions $\left(1 \% \mathrm{O}_{2}\right)$ were higher than those in the normoxic samples (GSE80140). In contrast, the gene expressions of PFKFB1 and PFKFB2 were not changed in hypoxic-cultured cells (Figure $1 \mathrm{~A}$ ). We also found that the gene expressions of $P F K F B 3$ and $P F K F B 4$ were increased in primary myeloma samples (GSE80545), while those of $P F K F B 1$ and $P F K F B 2$ were not changed (Figure 1B). Thus, in the myeloma cells, the gene expressions of $P F K F B 3$ and $P F K F B 4$ were induced under hypoxic conditions. Myeloma cell morphology and morphometry thus correlates with clinical stages and survival. Because MM includes three stages, which predict the myeloma's progression, we next investigated the gene expressions of PFKFB3 and PFKFB4 in the clinical stage. The GEO data (GSE13591) showed that the gene expressions of PFKFB3 and PFKFB4 were not changed in the clinical stage (Figure 1C).

\section{Changes in PFKFB3 and 4 expressions in myeloma cells under hypoxic conditions}

The GSE data indicated that the gene expressions of PFKFB3 and PFKFB4 were increased under hypoxic conditions. We next investigated the protein expressions of PFKFB3 and PFKFB4. Myeloma cells were cultured under $37^{\circ} \mathrm{C}, 5 \% \mathrm{CO}_{2}$, and $1 \% \mathrm{O}_{2}$ for $24 \mathrm{~h}$, after which immunoblot analysis was performed. We confirmed that the protein expressions of PFKFB3 and PFKFB4 were increased in MM cell lines (Figures $2 \mathrm{~A}, 2 \mathrm{C}$ ). We also confirmed that the gene expressions of PFKFB3 and PFKFB4 were increased during hypoxia by RT-PCR analysis (Figure 2B). Next, we performed the immunoblot analysis under hypoxia. We found that the expression of phospho-p38 MAPK was increased after 6- and 12-h under hypoxic conditions. The protein expressions of HIF1a and PFKFB3 were increased after 6-h to 24-h under hypoxic conditions. In contrast, PFKFB4 expression was increased after $24 \mathrm{~h}$ of hypoxic treatment (Figure 2D). We also confirmed that p38 MAPK phosphorylation was increased under hypoxia by ELISA analysis. The intracellular glucose level was not changed under hypoxia (Figure 2F), but the relative amount of LDH was increased (Figure 2G).

\section{Drug sensitivity and proliferation of myeloma cells under hypoxia}

Carfilzomib is a novel epoxyketone-based irreversible proteasome inhibitor. Carfilzomib demonstrates irreversible binding to the proteasome with minimal off-target inhibition of other proteases [17]. We next evaluated the anti-proliferative activity of carfilzomib in myeloma cells. The cells were cultured under $37^{\circ} \mathrm{C}, 5 \% \mathrm{CO}_{2}$, and $1 \% \mathrm{O}_{2}$ for $72 \mathrm{~h}$ or under normoxic conditions with the indicated concentration of carfilzomib. Treatment with carfilzomib under hypoxia for $72 \mathrm{~h}$ significantly decreased the sensitivity of carfilzomib in myeloma cells compared with treatment with the same dose of carfilzomib under normoxia 
(Figure 3A). Caspase 3/7 activities after carfilzomib treatment were also decreased under hypoxia (Figure 3B). Nuclear factor-kappa B (NF-kB) plays a key role in the survival and proliferation of many kinds of Bcell tumors, including MM [18]. We examined NF-kB activity under hypoxia. The phosphorylation of NF-kB was increased after $2 \mathrm{~h}$ in hypoxic culture conditions. The HIF1 a inhibitor, FM19G11, and p38 MAPK inhibitor, SB203580, were both found to inhibit the phosphorylation of NF-kB (Figure 3C). PFK158 is a potent and selective inhibitor of PFKFB3, and 5MPN is an inhibitor of PFKFB4. The activity of cell proliferation was reduced after PFK158 or 5MPN treatment in a dose-dependent manner (Figures 3D, 3E).

\section{PFKFB3 and PFKFB4 inhibitors enhance the activity of proteasome inhibitors in the myeloma cell line}

Because we found that the PFKFB3 inhibitor, PFK158 and PFKFB4 inhibitor, and 5MPN were effective on the myeloma cell line, we next investigated whether PFK158 or 5MPN could enhance the activity of the proteasome inhibitor carfilzomib. We found that PFK158 and 5MPN enhance carfilzomib sensitivity in hypoxic conditions (Supplemental Figure 1). The $\mathrm{Cl}$ provides a quantitative measure of the extent of drug interaction. Because $\mathrm{Cl}$ values were $<1$, these combination treatments were synergistic (data not shown). We next investigated the caspase $3 / 7$ activity when myeloma cells were co-treated with carfilzomib and PFK158 or 5MPN under hypoxic conditions. We observed elevated caspase 3/7 activity with co-treatment with carfilzomib and PFK158 or 5MPN (Figure 4A). We next examined the proteasome activity. We found that the 20 S proteasome activity was reduced by carfilzomib and PFK158 or 5MPN cotreatment (Figure 4B). Mitochondrial membrane potential is a key indicator of mitochondrial activity and is an essential component in the process of energy storage [19], so we evaluated it using the Mitochondria Staining Kit. The relative disrupted mitochondrial ratio was increased in carfilzomib and PFK158 or 5MPN co-treatment (Figure 4C). There are six human anti-apoptotic B-cell lymphoma 2 (BCL2) family proteins: $B C L-2$, B-cell lymphoma-extra large (BCL-XL), MCL-1, BCL-W, BFL-1, and BCL-2-like protein 10 (BCL2L10) [20]. BCL2L10 is predominantly expressed in normal human $B$ lymphocytes and is overexpressed in malignant plasma cells [21]. We found that the gene expression of BCL2L 10 was increased under hypoxic conditions by the GEO database. BCL2L10 expression was also correlated with PFKFB3 and PFKFB4 expression (Figure 4D). We next investigated protein expression by immunoblot analysis and found that the protein expressions of BCL-2, BCL-XL, and BCL2L10 were reduced after cotreatment with carfilzomib and PFK158 or 5MPN (Figure 4E). In contrast, the protein expression of NF-KB p65 was not changed compare to carfilzomib treatment.

\section{Activity of PFKFB3 and PFKFB4 inhibitors against bortezomib-resistant cells}

Bortezomib was approved for clinical use as a frontline treatment for newly diagnosed MM patients [22]. We investigated the efficacy of PFKFB3 and PFKFB inhibitors using the bortezomib-resistant cell line KMS-11/BTZ. The proliferation of KMS-11/BTZ was reduced by PFK158 or 5MPN in a dose-dependent manner (Figure 5A). We next examined the efficacy of the proteasome inhibitor and PFKFB3 or PFKFB inhibitor combination. Co-treatment with carfilzomib and PFK158 or 5MPN reduced cell proliferation compared with each drug alone (Figure 5B). Caspase $3 / 7$ activity was increased by co-treatment with carfilzomib and PFK158 or 5MPN (Figure 5C). Immunoblot analysis confirmed that the expressions of 
cleaved caspase 3 and cleaved PARP were increased by carfilzomib and PFK158 or 5MPN treatment (Figure 5D).

\section{Discussion}

Myeloma cells reside in the bone-marrow microenvironment, which is hypoxic [6]. Hypoxia is an imbalance between $\mathrm{O}_{2}$ supply and consumption that deprives cells or tissues of sufficient oxygen. In the gene expression profile, we observed that PFKFB3 and PFKFB4 expressions were increased under hypoxia. Metabolic reprogramming has been widely accepted as a hallmark of cancer [23]. In addition to promoting tumorigenesis, aerobic glycolysis plays a pivotal role in developing drug resistance [24]. In our study, we observed that the activity of carfilzomib was reduced under hypoxia. We observed that the activation of p38 MAPK was an early response to hypoxia, and that HIF1a expression was also increased. The inhibition of p38 MAPK and HIF1a prohibited NF-KB phosphorylation, which shows that p38 MAPK and HIF1a regulate NF-KB activity. NF-KB phosphorylation controls transcription in a gene-specific manner [25], and its increase in hypoxic conditions suggests that it may be related to the physiological processes, including cell proliferation, of the hypoxic condition. The combination of carfilzomib with PFK158 or 5MPN significantly decreased cell viability in vitro through activation of caspase 3/7. Mitochondria are essential regulators of cellular energy and metabolism [19] and have a crucial role in sustaining the growth and survival of cancer cells. The combination of carfilzomib with PFK158 or 5MPN significantly reduced mitochondrial membrane potential.

In our previous report, BCL2L10 was overexpressed in plasmocytes from MM patients but not in those from MGUS patients or normal healthy controls. BCL2L10 transgenic mice have been found to develop the characteristic features of human MM [26]. We found that the BCL-2 family gene, BCL2L10, was increased under hypoxia. We also demonstrated that the gene expression of $B C L 2 L 10$ was correlated with PFKFB3 and PFKFB4 expressions. These results indicate that BCL2L10, PFKFB3, and PFKFB4 may be related to myeloma progression.

The elevation of glycolysis enables sufficient production of glycolytic intermediates required for amino acid and nicotinamide adenine dinucleotide phosphate (NADPH) synthesis [27]. The role of enhanced glucose breakdown in carcinogenesis is well known because the attenuation of glycolysis inhibits the growth, and decreases the survival, of cancer cells. High PFKFB3/4 expression is significantly correlated with shorter overall survival in several cancers, such as cervical squamous cell carcinoma, colon cancer, glioma, melanoma, and neuroblastoma [28]. In a previous study, a high amount of PFKFB4 was found to indicate a poor prognosis in breast cancer because it is related to increased rates of metastasis [29].

During cancer progression, metabolic reprogramming is observed in parallel with alteration in the transcriptional profiles of cancer cells. Thus, PFKFB3 and PFKFB4 have been recognized as important metabolic targets in cancers. There have been several clinical trials of PFKFB3 inhibitors. Clinical trials assessing the safety of PFK158 were initiated in 2014, and no serious adverse events were reported [30]. Although the other PFKFB3 inhibitors, PFK15 and 3PO, have shown synergy with currently used 
antineoplastic agents in disrupting glycolysis and eliminating resistance mechanisms [10], it remains to be seen whether their efficacies in preclinical studies will translate into clinical trials.

In this study, we found that single treatment with the PFKFB3 inhibitor, PFK158 or PFKFB4 inhibitor, or $5 M P N$ decreased the proliferation of myeloma cells. Combination treatment with carfilzomib and PFK158 or 5MPN synergistically enhanced cell death, even under hypoxic conditions.

\section{Conclusions}

Tumor microenvironmental factors, such as hypoxia and the interaction between MM and bone marrow stromal cells (BMSCs), may become an important consideration for understanding disease progression and resistance to therapy. Our findings support the importance of PFKFB3 and PFKFB4 as target proteins in MM therapy under hypoxic conditions. The synergistic or sensitizing effect of PFKFB or PFKFB4 inhibitors with other chemotherapeutics, including carfilzomib, suggests that these compounds could represent important adjuvants or additives in future myeloma management strategies.

\section{Abbreviations}

PFKFB: 6-phosphofructo-2-kinase/fructose-2,6-bisphosphatase

HIF1a: Hypoxia-inducible factor 1a

MM: Multiple myeloma

PFK-2/FBPase-2: 6-phosphofructo-2-kinase/fructose-2,6-bisphosphatase

MGUS: monoclonal gammopathy of undetermined significance

p38 MAPK: p38 mitogen-activated protein kinase

ATP: adenosine triphosphate

FBS: fetal bovine serum

RT-PCR: real-time reverse transcription-polymerase chain reaction

GEO: Gene Expression Omnibus

NADPH: nicotinamide adenine dinucleotide phosphate

ELISA: enzyme-linked immunosorbent assay

NF-kB: nuclear factor-kappa B

Cl: combination index 
BCL-2: B-cell lymphoma 2

BCL-XL: B-cell lymphoma-extra large

BCL2L10: BCL-2-like protein 10

$\mathrm{O}_{2}$ : oxygen

$\mathrm{CO}_{2}$ : carbon dioxide

Abs: antibodies

\section{Declarations}

\section{Ethics approval and consent to participate}

Not applicable.

\section{Consent for publication}

Not applicable.

\section{Competing interests}

The authors declare that they have no competing interests.

\section{Funding}

This study was financially supported by a grant (20K07644) from the Japanese Ministry of Education, Culture, Sports, Science, and Technology (MEXT).

\section{Contributions}

SO, YT, and AG contributed to concept development and study design and wrote the manuscript. SO participated in the preparation of the figures. All authors read and approved the final manuscript.

\section{References}

1. Kumar SK, Rajkumar V, Kyle RA, van Duin M, Sonneveld P, Mateos MV, et al. Multiple myeloma. Nat Rev Dis Primers. 2017; 3:17046.

2. Du JS, Yen CH, Hsu CM, Hsiao HH. Management of myeloma bone lesions. Int J Mol Sci. 2021; 22: 3389.

3. Dhodapkar MV. MGUS to myeloma: A mysterious gammopathy of underexplored significance. Blood. 2016; 128: 2599-2606. 
4. Bringhen S, Mateos MV, Zweegman S, Larocca A, Falcone AP, Oriol A, et al. Age and organ damage correlate with poor survival in myeloma patients: Meta-analysis of 1435 individual patient data from 4 randomized trials. Haematologica. 2013; 98: 980-7.

5. Chan Chung KC, Tiedemann RE. Getting to the root of the problem: The causes of relapse in multiple myeloma. Expert Rev Anticancer Ther. 2014; 14: 251-4.

6. Zhang CC, Sadek HA. Hypoxia and metabolic properties of hematopoietic stem cells. Antioxid Redox Signal. 2014; 20: 1891-901.

7. Kierans SJ, Taylor CT. Regulation of glycolysis by the hypoxia-inducible factor (HIF): implications for cellular physiology. J Physiol. 2021; 599: 23-37.

8. Wu C, Khan SA, Peng LJ, Lange AJ. Roles for fructose-2,6-bisphosphate in the control of fuel metabolism: Beyond its allosteric effects on glycolytic and gluconeogenic enzymes. Adv Enzyme Regul. 2006; 46: 72-88.

9. Okar DA, Manzano A, Navarro-Sabatè A, Riera L, Bartrons R, Lange AJ. PFK-2/FBPase-2: Maker and breaker of the essential biofactor fructose-2,6-bisphosphate. Trends Biochem Sci. 2001; 26: 30-5.

10. Kotowski K, Rosik J, Machaj F, Supplitt S, Wiczew D, Jabłońska K, et al. Role of PFKFB3 and PFKFB4 in cancer: Genetic basis, impact on disease development/progression, and potential as therapeutic targets. Cancers (Basel). 2021; 13: 909.

11. Minchenko O, Opentanova I, Caro J. Hypoxic regulation of the 6-phosphofructo-2-kinase/fructose-2,6bisphosphatase gene family (PFKFB-1-4) expression in vivo. FEBS Lett. 2003; 554: 264-70.

12. Verduzco D, Lloyd M, Xu L, Ibrahim-Hashim A, Balagurunathan $Y$, Gatenby RA, et al. Intermittent hypoxia selects for genotypes and phenotypes that increase survival, invasion, and therapy resistance. PLoS One. 2015; 10: e0120958.

13. Chou TC. Drug combination studies and their synergy quantification using the Chou-Talalay method. Cancer Res. 2010; 70: 440-6.

14. Okabe S, Tanaka Y, Gotoh A. Targeting phosphoinositide 3-kinases and histone deacetylases in multiple myeloma. Exp Hematol Oncol. 2021; 10: 19.

15. Okabe S, Tanaka Y, Moriyama M, Gotoh A. Effect of dual inhibition of histone deacetylase and phosphatidylinositol-3 kinase in Philadelphia chromosome-positive leukemia cells. Cancer Chemother Pharmacol. 2020; 85: 401-412.

16. Yu L, Chen X, Wang L, Chen S. The sweet trap in tumors: Aerobic glycolysis and potential targets for therapy. Oncotarget. 2016; 7: 38908-38926. 
17. Khan ML, Stewart AK. Carfilzomib: A novel second-generation proteasome inhibitor. Future Oncol. 2011; 7: 607-12.

18. Demchenko YN, Brents LA, Li Z, Bergsagel LP, McGee LR, Kuehl MW. Novel inhibitors are cytotoxic for myeloma cells with NFkB inducing kinase-dependent activation of NFkB. Oncotarget. 2014; 5: 4554-66.

19. Zorova LD, Popkov VA, Plotnikov EY, Silachev DN, Pevzner IB, Jankauskas SS, et al. Mitochondrial membrane potential. Anal Biochem. 2018; 552: 50-59.

20. Adams JM, Cory S. The Bcl-2 protein family: Arbiters of cell survival. Science. 1998; 281: 1322-6.

21. Luciano F, Krajewska M, Ortiz-Rubio P, Krajewski S, Zhai D, Faustin B, et al. Nur77 converts phenotype of Bcl-B, an antiapoptotic protein expressed in plasma cells and myeloma. Blood. 2007; 109: 3849-55.

22. Chen D, Frezza M, Schmitt S, Kanwar J, Dou QP. Bortezomib as the first proteasome inhibitor anticancer drug: Current status and future perspectives. Curr Cancer Drug Targets. 2011; 11: 239-53.

23. Ward PS, Thompson CB. Metabolic reprogramming: A cancer hallmark even Warburg did not anticipate. Cancer Cell. 2012; 21: 297-308.

24. Altered glycolysis results in drug-resistant in clinical tumor therapy. Oncol Lett. 2021; 21: 369.

25. Christian F, Smith EL, Carmody RJ. The regulation of NF-KB subunits by phosphorylation. Cells. 2016; 5: 12 .

26. Hamouda MA, Jacquel A, Robert G, Puissant A, Richez V, Cassel R, et al. BCL-B (BCL2L10) is overexpressed in patients suffering from multiple myeloma (MM) and drives an MM-like disease in transgenic mice. J Exp Med. 2016; 213: 1705-22.

27. Yu L, Chen X, Wang L, Chen S. The sweet trap in tumors: Aerobic glycolysis and potential targets for therapy. Oncotarget. 2016; 7: 38908-38926.

28. Trojan SE, Markiewicz MJ, Leśkiewicz K, Kocemba-Pilarczyk KA. The influence of PFK-II overexpression on neuroblastoma patients' survival may be dependent on the particular isoenzyme expressed, PFKFB3 or PFKFB4. Cancer Cell Int. 2019; 19: 292.

29. Yao L, Wang L, Cao ZG, Hu X, Shao ZM. High expression of metabolic enzyme PFKFB4 is associated with poor prognosis of operable breast cancer. Cancer Cell Int. 2019; 19: 165.

30. Lu L, Chen Y, Zhu Y. The molecular basis of targeting PFKFB3 as a therapeutic strategy against cancer. Oncotarget. 2017; 8: 62793-62802.

\section{Figures}


Figure 1

A

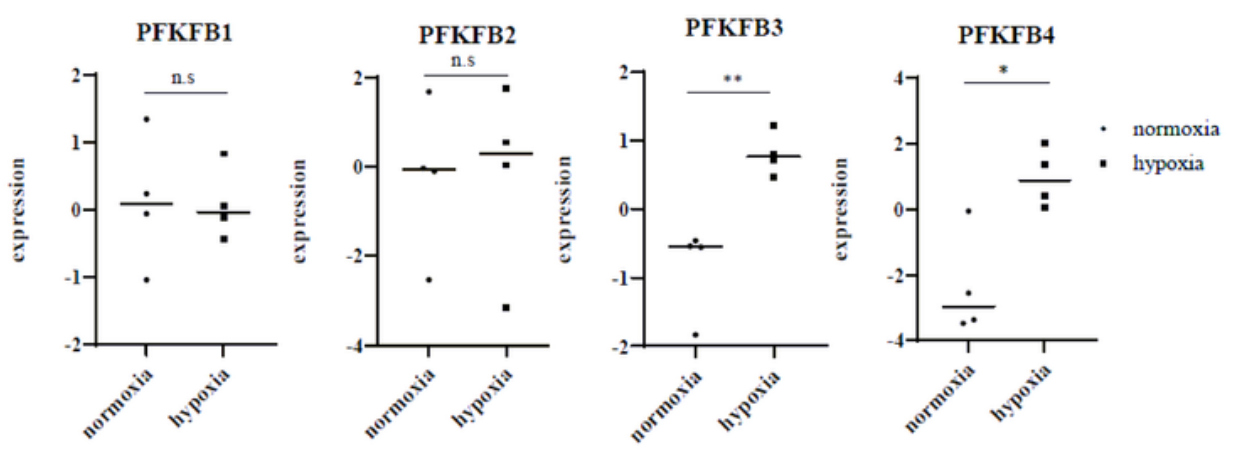

B

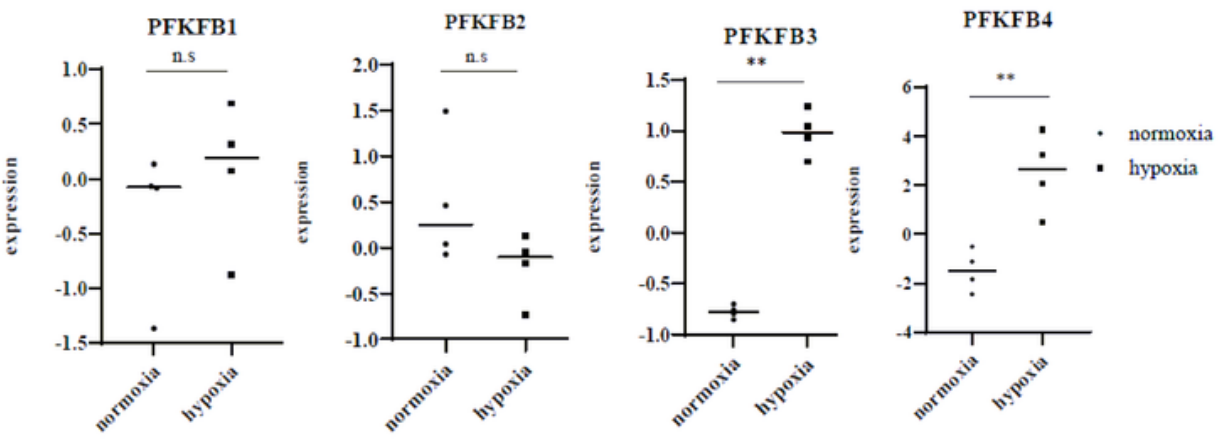

C

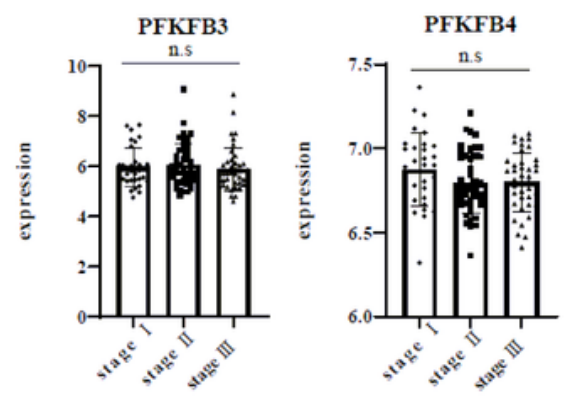

\section{Figure 1}

Gene expressions of PFKFB family members in the myeloma samples under hypoxic condition. (A) Gene expression profiles of the PFKFB family members (PFKFB1, PFKFB2, PFKFB3, and PFKFB4) were analyzed by comparing GEO data (GSE80140) for the normoxic $(n=4)$ and hypoxic groups $(n=4) .{ }^{*} p<0.05$, ${ }^{* *} p<0.01$ vs. normoxia. n.s.: not significant. (B) Gene expression profiles of the PFKFB family members against primary myeloma samples were analyzed by comparing GEO data (GSE80545) between the 
normoxic $(n=4)$ and hypoxic groups $(n=4) .{ }^{* *} p<0.01$ vs. normoxia. n.s.: not significant. (C) Gene expressions of PFKFB3 and PFKFB4 in the myeloma clinical staging system were analyzed by GSE13591. n.s.: not significant.

Figure 2

A
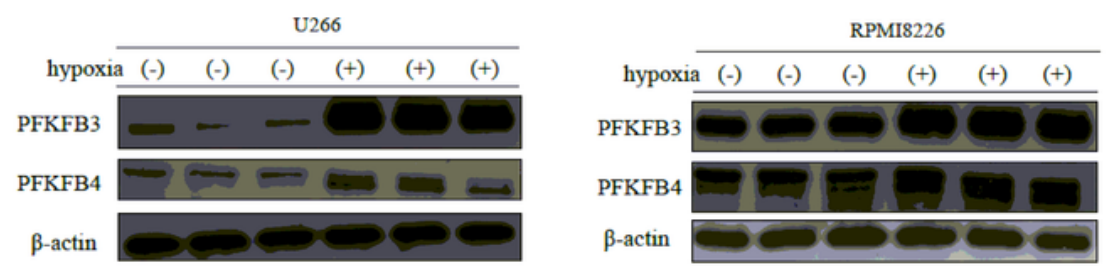

B
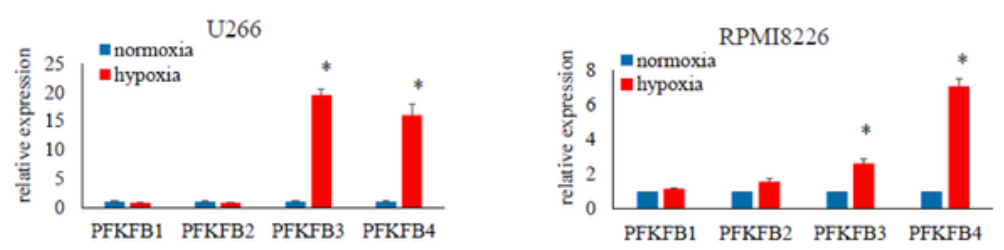

C

D
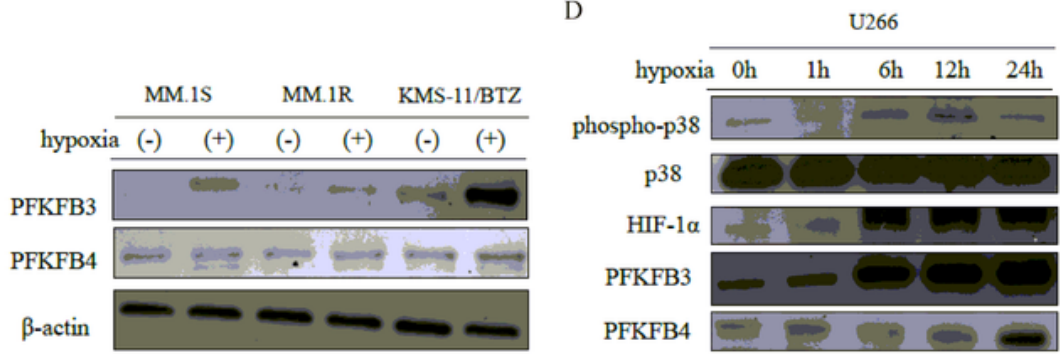
phospho-p38

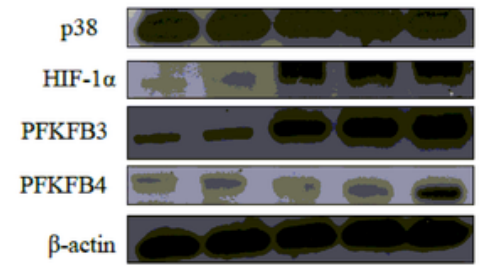

$\mathrm{E}$

F
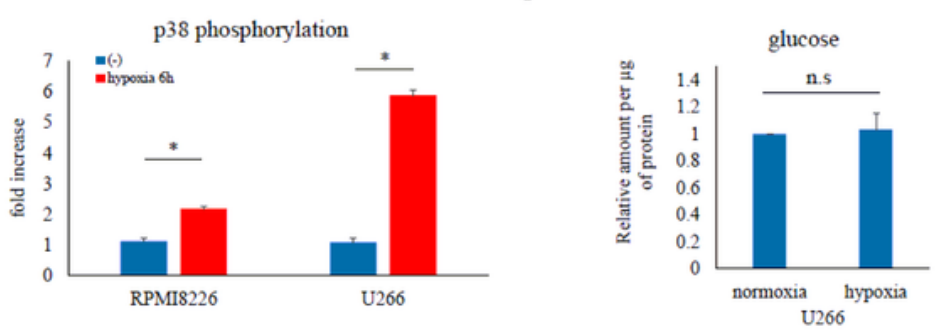

G

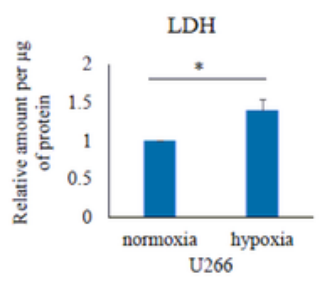

Figure 2

Expressions of PFKFB family members in the myeloma cells. (A, C) Myeloma cells (U266, RPMI8226, MM.1S, MM.1R, and KMS-11/BTZ) were cultured in RPMI 1640 medium under normoxia or hypoxia for 
24 h. PFKFB3 and PFKFB4 were examined using immunoblot analysis. Actin was the loading control. Results represent the mean of three independent experiments. (B) U266 and RPMI8226 were cultured under normoxia or hypoxia for $24 \mathrm{~h}$. Gene expressions of PFKFB family members were examined using quantitative RT-PCR analysis. Results represent three separate experiments. (D) U266 cells were cultured under hypoxia for the indicated amounts of times. Total extracts were examined by immunoblot analysis using antibodies against phospho-p38 MAPK (Thr180/Tyr182), p38 MAPK, HIF1a, PFKFB3, PFKFB4, and $\beta$-actin. (E) RPMI8226 and U266 cells were cultured under normoxia or hypoxia for $6 \mathrm{~h}$ and p38 MAPK activity was measured by the p38 MAPK (Phospho) [pT180/pY182] Multispecies InstantOne ${ }^{\text {TM }}$ ELISA Kit. $(F, G)$ U266 cells were cultured under normoxia or hypoxia for $24 \mathrm{~h}$. Intracellular glucose and LDH release were analyzed using the Glucose Assay Kit-WST and Cytotoxicity LDH Assay kit with water-soluble tetrazolium [WST] salt. ${ }^{*} \mathrm{p}<0.05 \mathrm{vs}$. normoxia or hypoxia treatment group. 
Figure 3

A
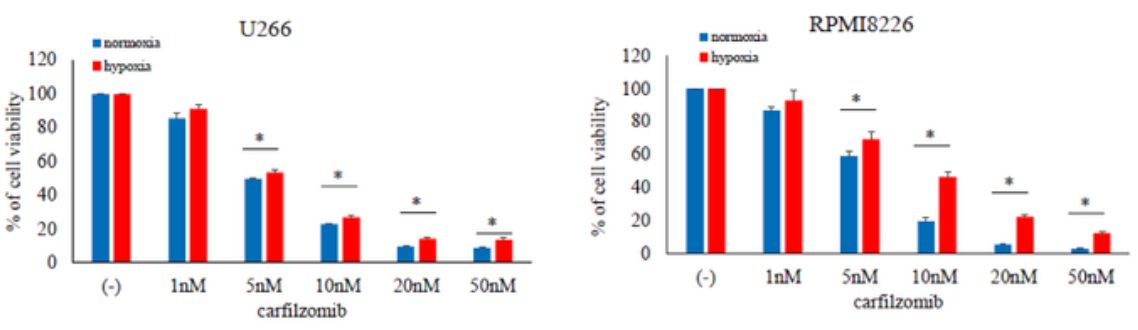

B

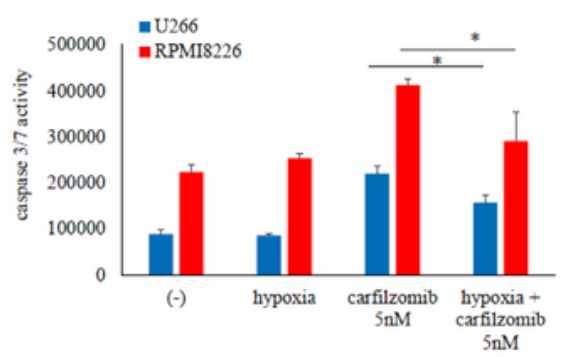

$\mathrm{C}$

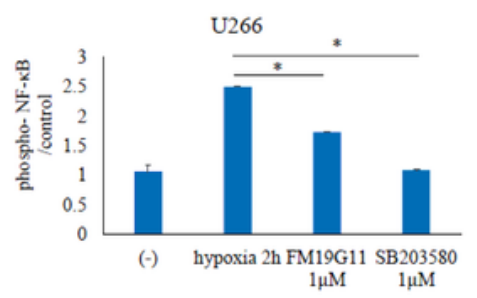

D
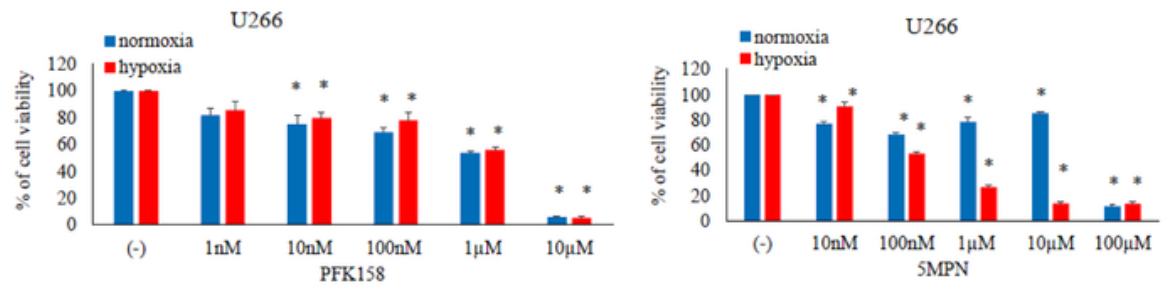

E
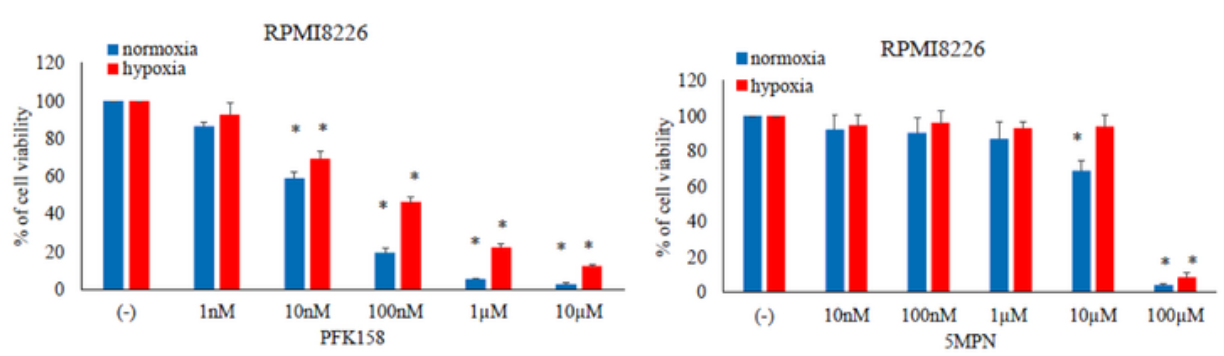

Figure 3

Effect of carfilzomib, PFK158, and 5MPN under normoxic and hypoxic conditions. (A) U266 and RPMI8226 cells were cultured under normoxia or hypoxia and incubated with the indicated concentration of carfilzomib for $72 \mathrm{~h}$. Cell growth was evaluated with the Cell Counting Kit-8. ${ }^{*}<<0.05$ vs. normoxia group. (B) U266 and RPMI8226 cells were cultured under normoxia or hypoxia and incubated with the indicated concentration of carfilzomib for $48 \mathrm{~h}$. Caspase $3 / 7$ activity was determined using the Caspase- 
Glo® 3/7 Assay System. Luminescence signals were measured using the Empire Multimode Plate Reader. (C) U266 cells were cultured under normoxia or hypoxia for $2 \mathrm{~h}$ and incubated with the indicated concentration of FM19G11 or SB203580. Phospho- and NF-kB were analyzed using the NF-kB p65 (Phospho) [pS536] Human InstantOne ${ }^{\text {TM }}$ ELISA Kit. (D, E) U266 and RPMI8226 cells were cultured under normoxia or hypoxia and incubated with the indicated concentration of PFK158 or 5MPN for $72 \mathrm{~h}$. Cell growth was evaluated with the Cell Counting Kit-8. ${ }^{\star} p<0.05$ vs. untreated cells.

Figure 4

A
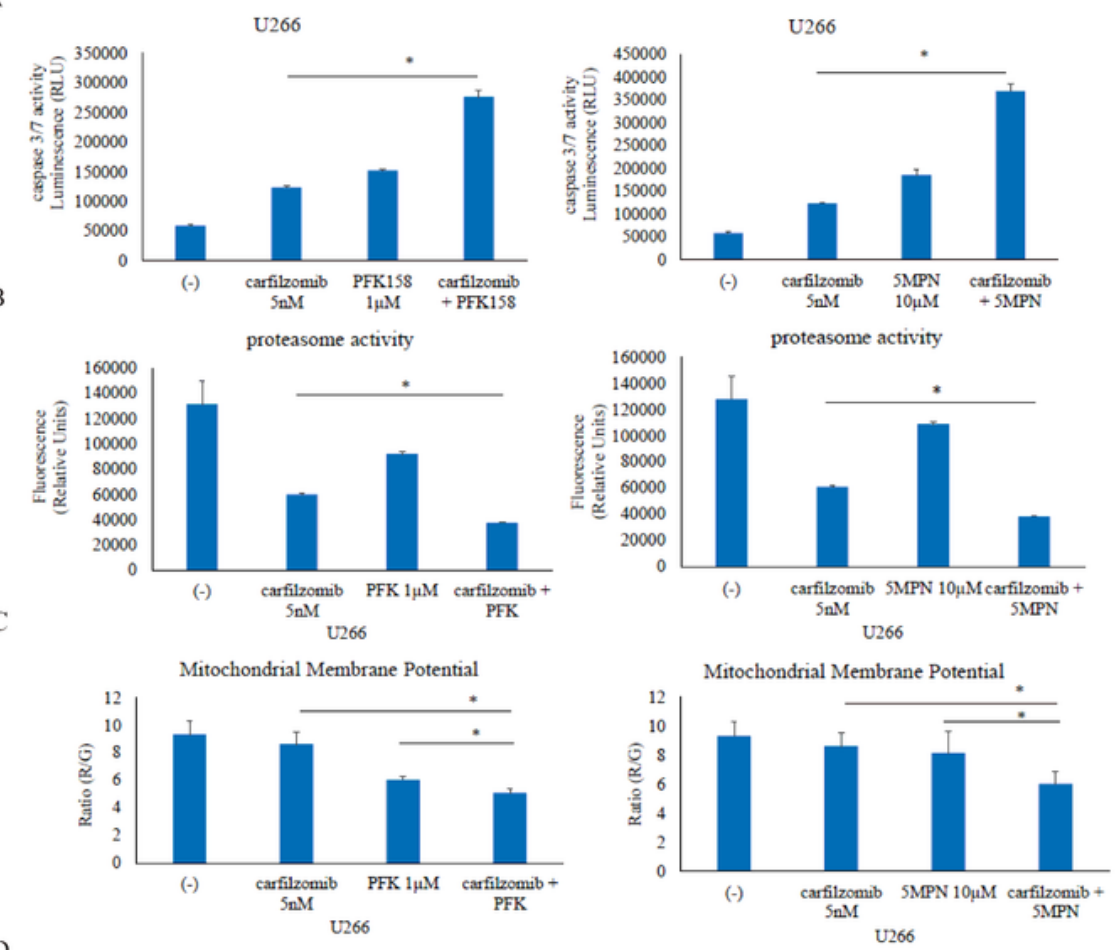

$\mathrm{D}$
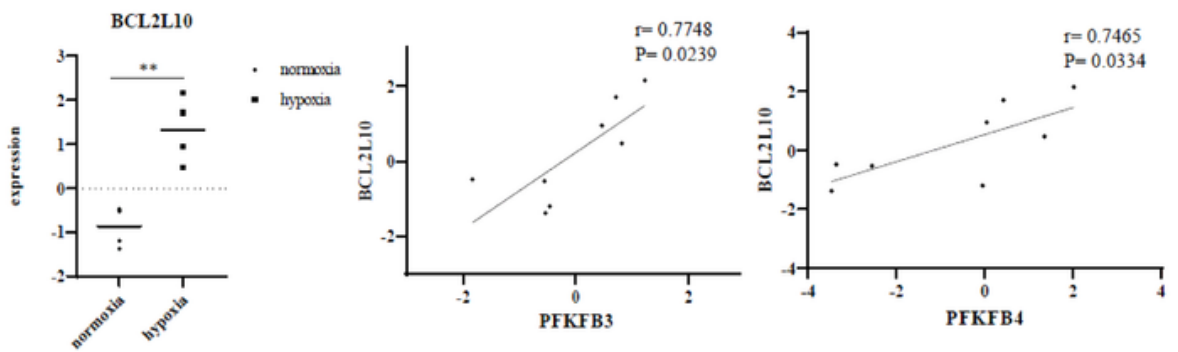

E
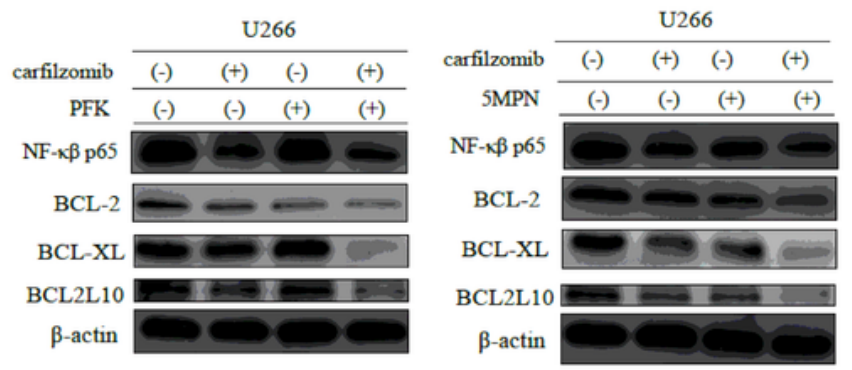
Co-treatment with carfilzomib and PFK158 or 5MPN induces cytotoxicity in myeloma cells under hypoxia. (A) U266 cells were treated with carfilzomib and/or PFK158 or 5MPN for $48 \mathrm{~h}$. Caspase 3/7 activity was determined using the Caspase-Glo ${ }^{8} 3 / 7$ Assay System. ${ }^{*} p<0.05$ vs. carfilzomib-treated cells. (B) U266 cells were treated with carfilzomib and/or PFK158 or 5MPN for $24 \mathrm{~h}$. A functional assay for detecting activity of the $20 \mathrm{~S}$ proteasome was examined by the $20 \mathrm{~S}$ Proteasome Assay Kit. ${ }^{*} p<0.05$ vs. carfilzomibtreated cells. (C) U266 cells were treated with carfilzomib and/or PFK158 or 5MPN for $24 \mathrm{~h}$. Mitochondrial membrane potentials were analyzed using the cationic JC-1 dye and the Mitochondria Staining Kit. ${ }^{*} \mathrm{p}<0.05$ vs. carfilzomib-treated cells. (D) The gene expression profile of BCL2L10 and the correlation between BCL2110 and PFKFB3 or PFKFB4 in the myeloma samples were analyzed by GEO data (GSE80140) ${ }^{* *} p<0.01$ vs. normoxia. (E) U266 cells were cultured under hypoxia for $24 \mathrm{~h}$ and incubated with the indicated concentration of carfilzomib and/or PFK158 or 5MPN. Total extracts were examined by immunoblot analysis using antibodies against NF-KB p65, BCL-2, BCL-XL, BCL2L10, and $\beta$-actin. 
Figure 5

A
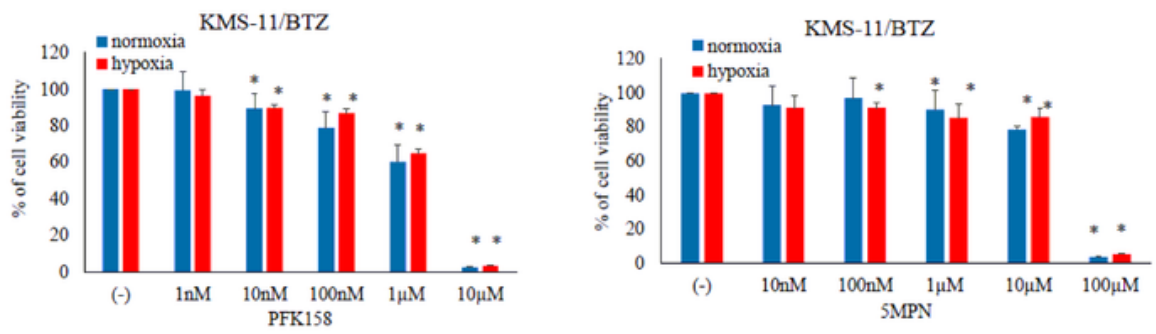

B
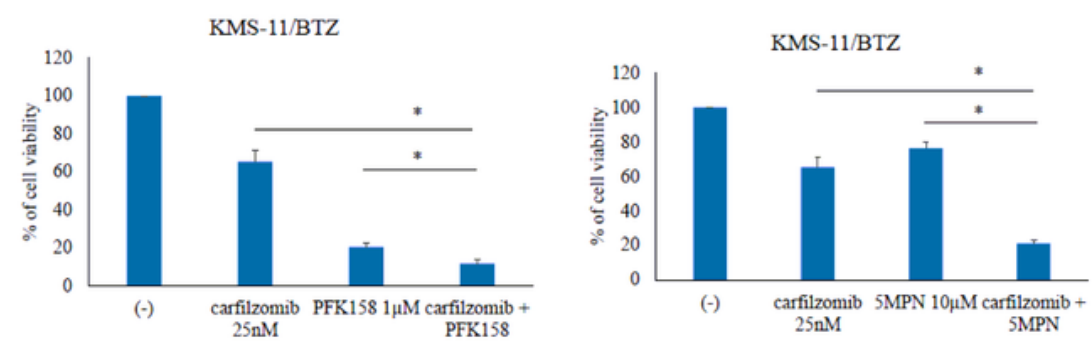

$\mathrm{C}$
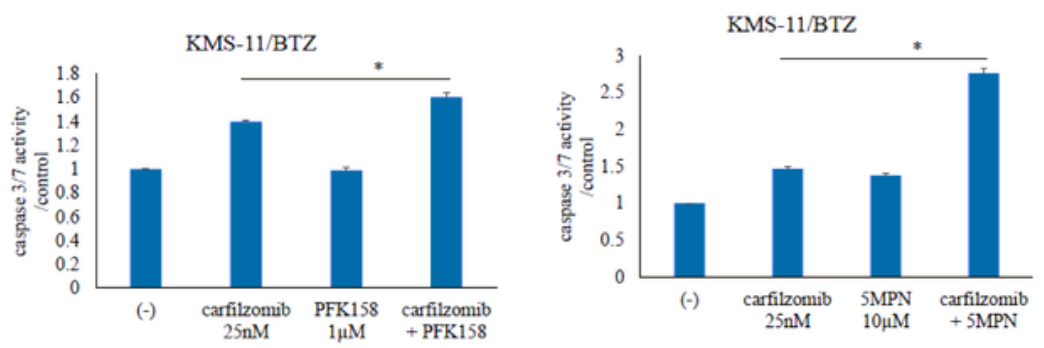

D

\begin{tabular}{|c|c|c|c|c|c|c|}
\hline \multirow[b]{2}{*}{ carfilzomib } & \multicolumn{6}{|c|}{ KMS-11/BTZ } \\
\hline & $(-)$ & $(+)$ & $(-)$ & $(+)$ & $(-)$ & $(+)$ \\
\hline PFK158 & $(-)$ & $(-)$ & $(+)$ & $(+)$ & $(-)$ & $(-)$ \\
\hline $5 \mathrm{MPN}$ & $(-)$ & $(-)$ & $(-)$ & $(-)$ & $(+)$ & $(+)$ \\
\hline cleaved PARP & & & & & & \\
\hline ved caspase 3 & & & & & & \\
\hline
\end{tabular}

Figure 5

Co-treatment with carfilzomib and PFK158 or 5MPN induces cytotoxicity in the bortezomib-resistant myeloma cells under hypoxia. (A) KMS-11/BTZ cells were cultured under normoxia or hypoxia and incubated with the indicated concentration of PFK158 or 5MPN for $72 \mathrm{~h}$. Cell growth was evaluated with the Cell Counting Kit-8. ${ }^{*} \mathrm{p}<0.05$ vs. untreated cells. (B) KMS-11/BTZ cells were treated with carfilzomib and/or PFK158 or 5MPN for $72 \mathrm{~h}$. Cell growth was evaluated with the Cell Counting Kit-8. ${ }^{*} \mathrm{p}<0.05$ vs. 
carfilzomib or PFK158- or 5MPN-treated cells. (C) KMS-11/BTZ cells were treated with carfilzomib and/or PFK158 or 5MPN for $48 \mathrm{~h}$. Caspase $3 / 7$ activity was determined using the Caspase-Glo ${ }^{\circledR} 3 / 7$ Assay System. ${ }^{*} p<0.05$ vs. carfilzomib-treated cells. (D) KMS-11/BTZ cells were cultured under hypoxia for $24 \mathrm{~h}$ and incubated with the indicated concentration of carfilzomib and/or PFK158 or 5MPN. Total extracts were examined by immunoblot analysis using antibodies against cleaved caspase 3 , cleaved PARP, and $\beta$-actin.

\section{Supplementary Files}

This is a list of supplementary files associated with this preprint. Click to download.

- SupplementalFigure202111.tif 